\title{
Announcement Breast Cancer Research non-peer-review research depository
}

Published: 17 December 1999

The tradition of circulating research findings before peerreview is old and continues today. In addition to the informal circulation of non-peer-reviewed research reports between scientists, there are also well-organised schemes, of which the most established currently is the Internet-based preprint repository in physics run from the Los Alamos Institute (http://arxiv.org/). The National Institutes of Health (NIH) also offered a successful and well regarded preprint service in the 1960 s.

There is currently a declared plan from the NIH that invites any journal that wishes to participate to deposit non-peerreviewed research (preprints) into PubMed Central - the new central repository for biomedical research run by the $\mathrm{NIH}$ (http://www.nih.gov/welcome/director/ebiomed/ebi.htm). The Breast Cancer Research journal reflects the belief that a facility for depositing non-peer-reviewed research data can bring major benefits for the dissemination of research findings, and we will support and promote the availability of this service.

There are two main situations where the deposition of non-peer-review research can be especially valuable:

1. Researchers often have findings which are not currently published partly because of the space restrictions imposed by traditional journals, and partly because no tradition has developed about how to handle particular kinds of information. These may nonetheless be findings that could usefully add to scientific knowledge, even sometimes in a minor or marginal way. Examples of such findings include:

- improvements in techniques

- detailed laboratory protocols

- design of experiments

- unexpected observations

- preclinical work on drug development

- design of clinical trials

- genome data

- microarrays

- DNA sequences
Breast Cancer Res 2000, 2:1

(C) Current Science Ltd

2. Deposition of primary research reports as a first stage in the peer-review process. This method to some extent replicates the informal way in which research articles are circulated among scientists at present, often prior to publication. Authors have always circulated their research in this way in order to generate comments, suggestions and critiques from informed colleagues. Depositing such information in public places will broaden the community who can access this information and offer comments. It may also help to establish genuine priority and make information available earlier without the often considerable delays that may occur as a result of the reviewing process.

Both these benefits, and others that may become more apparent in the future, will, we believe, be of value to the scientific community in general.

At the same time we are aware that the development of the non-peer-reviewed public deposition of research findings may bring some problems as well as the expected benefits. As publishers we will be sensitive to the potential dangers and we will respond quickly when problems occur. As with all new endeavours, we very much depend on all our readers who we hope will both participate in the newly available services and give us feedback.

Facilities for depositing research findings will be made available in the Breast Cancer Research website (http://breast-cancer-research.com), with full instructions about how to use this new service.
Anne Greenwood Managing Director Current Science Ltd 